\title{
Comparative Study of Generalized Sum Graphs via Degree-Based Topological Indices
}

\author{
Muhammad Javaid $\left(D,{ }^{1}\right.$ Saira Javed, ${ }^{1}$ and Ebenezer Bonyah ${ }^{2}{ }^{2}$ \\ ${ }^{1}$ Department of Mathematics, School of Science, University of Management and Technology, Lahore 54 770, Pakistan \\ ${ }^{2}$ Department of Mathematics Education, Akenten Appiah-Menka University of Skills Training and Entrepreneurial Development, \\ Kumasi 00233, Ghana \\ Correspondence should be addressed to Ebenezer Bonyah; ebbonya@gmail.com
}

Received 17 September 2021; Accepted 3 January 2022; Published 14 February 2022

Academic Editor: Muhammad Imran

Copyright (c) 2022 Muhammad Javaid et al. This is an open access article distributed under the Creative Commons Attribution License, which permits unrestricted use, distribution, and reproduction in any medium, provided the original work is properly cited.

\begin{abstract}
In theoretical chemistry, topological indices (TIs) have important role to predict various physical and structural properties of the study under molecular graphs. Among all topological indices, Zagreb-type indices have been used more effectively in the chemical literature. In this paper, we have computed first Zagreb, second Zagreb, forgotten, and hyper Zagreb indices of the generalized Q-sum graph $\left(H_{1_{\left(Q_{\alpha}\right)}}\left[H_{2}\right]\right)$ in the form of different TIs of its basic graphs, where $\alpha \geq 1$ is a positive integer. This family of graphs is obtained by the lexicographic product of the graph $Q_{\alpha}\left(H_{1}\right)$ and $H_{2}$, where $Q_{\alpha}\left(H_{1}\right)$ is constructed with the help of the generalized line superposition operation $Q_{\alpha}$ on $H_{1}$. As a conclusion, we also checked the correlation between predefined graph $H_{1}\left[H_{2}\right]$ under the operation of lexicographic product $H_{1}$ and $H_{2}$ with newly defined generalized Q-sum graphs $\left(H_{1_{\left(Q_{\alpha}\right)}}\left[H_{2}\right]\right)$ using linear regression models of various degree-based TIs.
\end{abstract}

\section{Introduction and Preliminaries}

In the computing analysis of chemical compounds, the chemical structural formulas are usually represented by graphs. Mathematically, for a graph $H$, the vertex and edge sets are denoted by $V(H)$ and $E(H) \subseteq V(H) \times V(H)$, respectively. The degree of the vertex $z \in V(H)$ (denoted by $d(z))$ is the number of incident edges on it. The distance between any two vertices $z$ and $y$ in $V(H)$ is denoted by $d(z, y)$ and defined as the number of edges in a shortest path existing between the vertices $z$ and $y$. Thoroughly, the graph $H$ is considered as a finite and simple graph.

The subject of chemical graph theory has many applications in chemistry. In a chemical graph, a vertex and an edge correspond to an atom and a chemical bond between them, respectively. A topological index is a function which presents a chemical graph in the form of a numerical number that is used to model the chemical and physical properties of molecules in quantitative structure property and activity relationships.
For a molecular graph $H$, the first Zagreb index $M_{1}(H)$ and second Zagreb index $M_{2}(H)$ are firstly considered by Gutman and Trinajstic [1] in 1972 to study the total $\pi$ electron energy of the molecular graph $H$ which are defined as follows:

$$
\begin{aligned}
& M_{1}(H)=\sum_{z \in V(H)} d^{2}(z)=\sum_{z y \in E(H)}[d(z)+d(y)], \\
& M_{2}(H)=\sum_{z y \in E(H)}[d(z) \times d(y)] .
\end{aligned}
$$
[2]:

In 2015, forgotten index ( $F$ index) is defined as follows

$$
F(H)=\sum_{z \in V(H)} d^{3}(z)=\sum_{z y \in E(H)}\left[d^{2}(z)+d^{2}(y)\right] .
$$

In 2005, the first general Zagreb index is defined as follows [3]: 


$$
M_{1}^{\beta+1}(H)=\sum_{z \in V(H)} d^{\beta+1}(z)=\sum_{z y \in E(H)}\left[d^{\beta}(z)+d^{\beta}(y)\right] .
$$

The concept of the general randic index (GRI) is defined by Bollobas and Erdos as

$$
R_{\beta}(H)=\sum_{z y \in E(H)}[d(z) \times d(y)]^{\beta} .
$$

In 2013, hyper Zagreb index is defined by Shirdel et al. as follows [4]:

$$
\operatorname{HM}(H)=\sum_{z y \in E(H)}[d(z)+d(y)]^{2}
$$

For more studies, we refer to [5]. In particular, we can find the results of TIs for various families of graphs such as nanosheets \& nanostars dendrimers [6, 7], hex-derived networks [8], benzene networks $[9,10]$, and cellulose networks [11]. In addition, for the studies of the complex graphs, operations on graphs play a key role, where the original graphs are called the factors of the newly constructed graph under operations [12].

Yan et al. [13] defined the line superposition operation $Q_{1}$ related to the subdivision of $H$ and computed the Wiener index of this derived graph $Q_{1}(H)$, where $Q_{1}(H)$ is obtained by inserting one new vertex in every edge of $H$ and joining those pairs of new vertices by edges which have common adjacent (original) vertices (see Figure 1). Liu et al. extended this operation for any integral value of $\alpha \geq 1$ and obtained the generalized line superposition graph $Q_{\alpha}(H)$ from the graph $H$ by inserting $\alpha$ vertices in each edge and joining those pairs of new vertices by edges which have common adjacent (original) vertices [14] (see Figures 2 and 3).

Taeri et al. [15] constructed the $Q_{1}$-sum graph by Cartesian product of $Q_{1}\left(H_{1}\right)$ and $H_{2}$, where $Q_{1}\left(H_{1}\right)$ is a line superposition graph, and $H_{1}$ and $H_{2}$ are assumed to be two connected graphs. Also, they computed the Wiener index of $Q_{1}$-sum graph. Whereas, Chu et al. [16], Deng et al. [17], Akhter et al. [18], and Liu et al. [14] calculated the different indices of $Q_{1}$-sum graph $\left(H_{1_{\left(Q_{1}\right)}}+H_{2}\right)$ based on the Cartesian product. Recently, Liu et al. [19] extended this operation for any integral value of $k$ and obtained the generalized line superposition graph $Q_{k}\left(H_{1}\right)$ of the graph $H_{1}$. Moreover, they constructed the generalized Q-sum graph $\left(H_{1_{\left(Q_{k}\right)}}+H_{2}\right)$ and computed their Zagreb indices. Javaid et al. [20] constructed the generalized Q-sum graph based on strong product and computed its first and second Zagreb indices.

The composition or lexicographic product of two connected graphs $H_{1}$ and $H_{2}$, which is denoted by $H_{1}\left[H_{2}\right]$, is a graph such that the set of vertices is $V\left(H_{1}\right) \times V\left(H_{2}\right)$, and two vertices $\left(z_{1}, y_{1}\right)$ and $\left(z_{2}, y_{2}\right)$ will be adjacent in $H_{1}\left[H_{2}\right]$ if $\left[z_{1}=z_{2}\right.$, and $y_{1}$ is adjacent to $\left.y_{2}\right]$ or $\left[z_{1}\right.$ is adjacent to $z_{2}$, and $y_{1}$ is adjacent to $y_{2}$ ].

Sarala et al. [21], De [22], Pattabiraman [23], Pattabiraman and Santhakumar [24], and Suresh and Devi [25] have computed different degree-related Zagreb indices for the graphs based online superposition operation and lexicographic product.

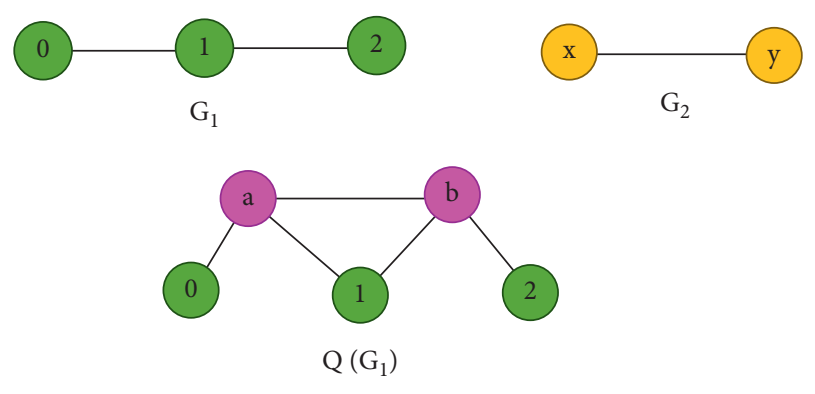

FIgURE 1: Line superposition operation for $P_{3}$ denoted by $Q_{1}\left(P_{3}\right)$.

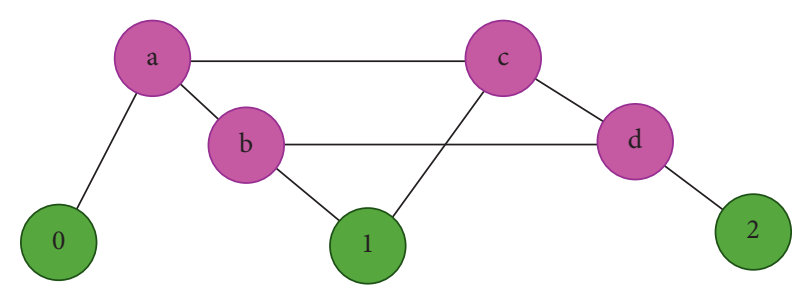

FIgURE 2: Generalized line superposition operation for $P_{3}$ and $\alpha=2$ denoted by $Q_{2}\left(P_{3}\right)$.

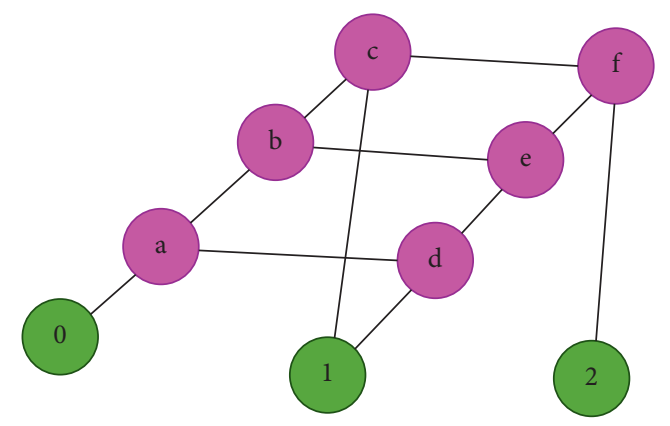

FIgURe 3: Generalized line superposition operation for $P_{3}$ for $\alpha=3$ denoted by $Q_{3}\left(P_{3}\right)$.

(1) $Q_{\alpha}$-sum: let $H_{1}$ and $H_{2}$ be two graphs; first, we apply generalized line superposition operation on $H_{1}$ to get $Q_{\alpha}\left(H_{1}\right)$ graph. Then, we take lexicographic product between $Q_{\alpha}\left(H_{1}\right)$ and $H_{2}$ to get $Q_{\alpha}$-Sum graph denoted by $H_{1_{\left(Q_{\alpha}\right)}}\left[H_{2}\right]$. $Q_{\alpha}$-sum graph having vertex-set

$$
\begin{aligned}
V\left(H_{1_{\left(Q_{\alpha}\right)}}\left[H_{2}\right]\right) & =V\left(Q_{\alpha}\left(H_{1}\right)\right) \times V\left(H_{2}\right) \\
& =\left(V\left(H_{1}\right) \cup \alpha\left(E\left(H_{1}\right)\right)\right) \times V\left(H_{2}\right),
\end{aligned}
$$

such that two vertices $\left(z_{1}, y_{1}\right)$ and $\left(z_{2}, y_{2}\right)$ of $V\left(H_{1_{\left(Q_{\alpha}\right)}}\left[H_{2}\right]\right)$ are adjacent if $\left[z_{1}=z_{2}\right.$ in $V\left(H_{1}\right)$, and $y_{1}$ is adjacent to $y_{2}$ in $\left.E\left(H_{2}\right)\right]$ or $\left[z_{1}\right.$ is adjacent to $z_{2}$ in $E\left(Q_{\alpha}\left(H_{1}\right)\right)$, and $y_{1}$ is adjacent to $y_{2}$ in $E\left(H_{2}\right)$ ], where $\alpha \geq 1$ is a positive integer (see Figure 4).

To check the correlation between the predefined lexicographic product of two simple graphs $\left(H_{1}\left[H_{2}\right]\right)$ and newly defined $Q_{\alpha}$-sum graphs $\left(H_{1_{\left(Q_{\alpha}\right)}}\left[H_{2}\right]\right)$, one may use the linear regression when having a linear relationship between the dependent variable $\left(X=H_{1}\left[H_{2}\right]\right)$ and the independent variable $\left(Y=H_{1_{\left(Q_{\alpha}\right)}}\left[H_{2}\right]\right)$. For further studies related to operations and graph products, the readers are referred to 


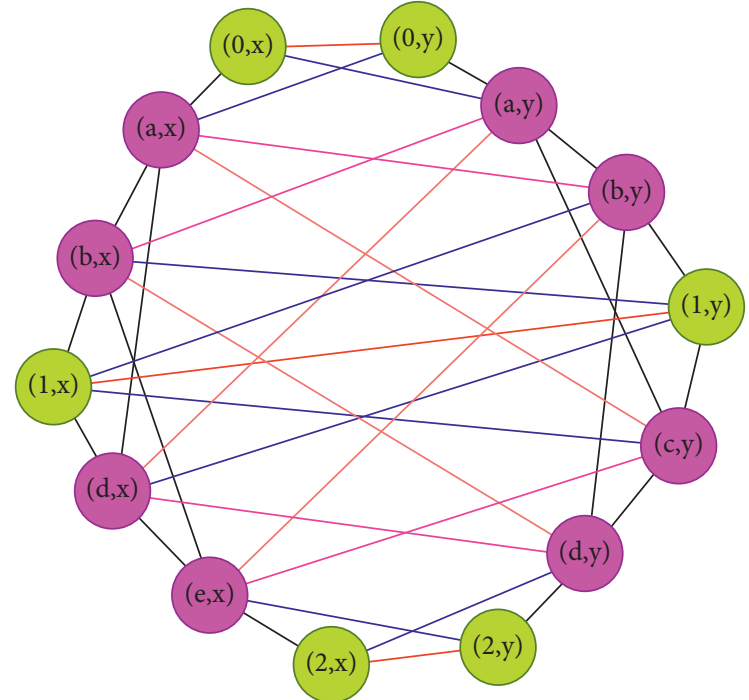

Figure 4: Generalized $Q_{\alpha}$-sum graph $H_{1_{\left(Q_{\alpha}\right)}}\left[H_{2}\right]$ for $P_{3_{\left(Q_{3}\right)}}\left[P_{2}\right]$. superposition graph $Q_{\alpha}\left(H_{1}\right)$ from the graph actual graph $H_{1}$. Then, we have constructed a $Q_{\alpha}$-sum graph based on the lexicographic product of $Q_{\alpha}\left(H_{1}\right)$ and $H_{2}$, denoted by $H_{1_{\left(O_{0}\right)}}\left[H_{2}\right]$. Moreover, we have computed the first Zagreb, second Zagreb, forgotten, and hyper Zagreb indices of $H_{\left.1_{(}\right)}\left[H_{2}\right]$ in terms of its factor graphs $H_{1}$ and $H_{2}$. We have extended this work by constructing linear regression models between $\left(H_{1}\left[H_{2}\right]\right)$ and $Q_{\alpha}$-sum graphs $\left(H_{1_{\left(Q_{\alpha}\right)}}\left[H_{2}\right]\right)$ via foresaid degree-based TIs.

\section{Main Results}

This section covers the main results.

Theorem 1. Let $H_{1}$ and $H_{2}$ be two connected graphs such that $\left|V\left(H_{1}\right)\right|,\left|V\left(H_{2}\right)\right| \geq 4$. For $\alpha \geq 1$,

[26-29]. In this paper, we have used the generalized line superposition operation to construct a new generalized line

$$
\begin{aligned}
M_{1}\left(H_{1_{\left(Q_{\alpha}\right)}}\left[H_{2}\right]\right)= & 8\left|V\left(H_{2}\right)\right|\left|E\left(H_{1}\right)\right|\left|E\left(H_{2}\right)\right|+3\left|V\left(H_{2}\right)\right|^{3} M_{1}\left(H_{1}\right)+\left|V\left(H_{1}\right)\right| M_{1}\left(H_{2}\right) \\
& +2(\alpha-1)\left|V\left(H_{2}\right)\right|^{2} M_{1}\left(H_{1}\right)+(\alpha)\left|V\left(H_{2}\right)\right|^{3}\left[M_{3}\left(H_{1}\right)+2 M_{2}\left(H_{1}\right)-2 M_{1}\left(H_{1}\right)\right] .
\end{aligned}
$$

Proof.

$$
\begin{aligned}
M_{1}\left(H_{1_{\left(Q_{\alpha}\right)}}\left[H_{2}\right]\right) & \left.=\sum_{\left(z_{1}, y_{1}\right)\left(z_{2}, y_{2}\right) \in E\left(H_{1}\left(Q_{\alpha}\right)\right.}\left[H_{2}\right]\right) \\
& =\sum_{z \in V\left(H_{1}\right)} \sum_{y_{1} y_{2} \in E\left(H_{2}\right)}\left[d\left(z, y_{1}\right)+d\left(z, y_{2}\right)\right]+\sum_{y_{1} \in V\left(H_{2}\right)} \sum_{y_{2} \in V\left(H_{2}\right)} \sum_{z_{1} z_{2} \in E\left(Q_{\alpha}\left(H_{2}\right)\right)}\left[d\left(z_{2}, y_{2}\right)\right] \\
& =\sum_{A}+\sum_{B} .
\end{aligned}
$$

Now,

$$
\begin{aligned}
\sum_{A} & =\sum_{z \in V\left(H_{1}\right)} \sum_{y_{1} y_{2} \in E\left(H_{2}\right)}\left[d\left(z, y_{1}\right)+d\left(z, y_{2}\right)\right]=\sum_{z \in V\left(H_{1}\right)} \sum_{y_{1} y_{2} \in E\left(H_{2}\right)}\left[2\left|V\left(H_{2}\right)\right| d_{H_{1}}(z)+d_{H_{2}}\left(y_{1}\right)+d_{H_{2}}\left(y_{2}\right)\right] \\
& =4\left|V\left(H_{2}\right)\left\|E\left(H_{1}\right)\right\| E\left(H_{2}\right)\right|+\left|V\left(H_{1}\right)\right| M_{1}\left(H_{2}\right), \\
\sum_{B} & =\sum_{y_{1} \in V\left(H_{2}\right)} \sum_{y_{2} \in V\left(H_{2}\right)} \sum_{z_{1} z_{2} \in E\left(Q_{\alpha}\left(H_{1}\right)\right)}\left[d\left(z_{1}, y_{1}\right)+d\left(z_{2}, y_{2}\right)\right]
\end{aligned}
$$




$$
\begin{aligned}
& =\sum_{y_{1} \in V\left(H_{2}\right)} \sum_{y_{2} \in V\left(H_{2}\right)} \sum_{\substack{z_{1} z_{2} \in E\left(Q_{\alpha}\left(H_{1}\right)\right) \\
z_{1} \in V\left(H_{1}\right) \\
z_{2} \in V\left(Q_{\alpha}\left(H_{1}\right)\right)-V\left(H_{1}\right)}}\left[d\left(z_{1}, y_{1}\right)+d\left(z_{2}, y_{2}\right)\right]+\sum_{y \in V\left(H_{2}\right)} \sum_{\substack{z_{1} z_{2} \in E\left(Q_{\alpha}\left(H_{1}\right)\right) \\
z_{1} z_{2} \in V\left(Q_{\alpha}\left(H_{1}\right)\right)-V\left(H_{1}\right)}}\left[d\left(z_{1}, y\right)+d\left(z_{2}, y\right)\right] \\
& +\sum_{y_{1} \in V\left(H_{2}\right)} \sum_{y_{2} \in V\left(H_{2}\right)} \sum_{\substack{z_{1} z_{2} \in E\left(Q_{\alpha}\left(H_{1}\right)\right) \\
z_{1} z_{2} \in V\left(Q_{\alpha}\left(H_{1}\right)\right)-V\left(H_{1}\right)}}\left[d\left(z_{1}, y_{1}\right)+d\left(z_{2}, y_{1}\right)\right]=\sum_{B_{1}}+\sum_{B_{2}}+\sum_{B_{3}} \\
& \sum_{B_{1}}=\sum_{y_{1} \in V\left(H_{2}\right)} \sum_{y_{2} \in V\left(H_{2}\right)} \sum_{\substack{z_{1} z_{2} \in E\left(Q_{\alpha}\left(H_{1}\right)\right) \\
z_{1} \in V\left(H_{1}\right) \\
z_{2} \in V\left(Q_{\alpha}\left(H_{1}\right)\right)-V\left(H_{1}\right)}}\left[d\left(z_{1}, y_{1}\right)+d\left(z_{2}, y_{2}\right)\right] \\
& =\sum_{y_{1} \in V\left(H_{2}\right)} \sum_{y_{2} \in V\left(H_{2}\right)} \sum_{\substack{z_{1} z_{2} \in E\left(Q_{\alpha}\left(H_{2}\right)\right) \\
z_{1} \in V\left(H_{1}\right) \\
z_{2} \in V\left(Q_{\alpha}\left(H_{1}\right)\right)-V\left(H_{1}\right)}}\left[\left|V\left(H_{2}\right)\right| d_{Q_{\alpha}\left(H_{1}\right)}\left(z_{1}\right)+d_{H_{2}}\left(y_{1}\right)+\left|V\left(H_{2}\right)\right| d_{Q_{\alpha}\left(H_{1}\right)}\left(z_{2}\right)\right] \\
& =\sum_{y_{1} \in V\left(H_{2}\right)} \sum_{y_{2} \in V\left(H_{2}\right)} \sum_{\substack{z_{1} z_{2} \in E\left(Q_{\alpha}\left(H_{2}\right)\right) \\
z_{1} \in V\left(H_{1}\right) \\
z_{2} \in V\left(Q_{\alpha}\left(H_{1}\right)\right)-V\left(H_{1}\right)}}\left[\left|V\left(H_{2}\right)\right| d_{H_{1}}\left(z_{1}\right)+d_{H_{2}}\left(y_{1}\right)\right] \\
& +\sum_{y_{1} \in V\left(H_{2}\right)} \sum_{y_{2} \in V\left(H_{2}\right)} \sum_{\substack{z_{1} z_{2} \in E\left(Q_{\alpha}\left(H_{2}\right)\right) \\
z_{1} \in V\left(H_{1}\right) \\
z_{2} \in V\left(Q_{\alpha}\left(H_{1}\right)\right)-V\left(H_{1}\right)}}\left[\left|V\left(H_{2}\right)\right| d_{Q_{\alpha}\left(H_{1}\right)}\left(\left(z_{2}\right)\right)\right] \\
& =4\left|V\left(H_{2}\right) \| E\left(H_{1}\right)\right|\left|E\left(H_{2}\right)\right|+\left|V\left(H_{2}\right)\right|^{3} M_{1}\left(H_{1}\right) \\
& +\sum_{y_{1} \in V\left(H_{2}\right)} \sum_{y_{2} \in V\left(H_{2}\right)} \sum_{\substack{z_{1} z_{2} \in E\left(Q_{\alpha}\left(H_{1}\right)\right) \\
z_{1} \in V\left(H_{1}\right) \\
z_{2} \in V\left(Q_{\alpha}\left(H_{1}\right)\right)-V\left(H_{1}\right)}}\left[\left|V\left(H_{2}\right)\right| d_{Q_{\alpha}\left(H_{1}\right)}\left(z_{2}\right)\right] .
\end{aligned}
$$

For $z_{2} \in V\left(Q_{\alpha}\left(H_{1}\right)\right)-V\left(H_{1}\right)$ which is inserted into the edge $u v$ of $\left(H_{1}\right)$, we have $d_{Q_{\alpha}\left(H_{1}\right)}\left(z_{2}\right)=d_{H_{1}}(u)+d_{H_{1}}(v)$.

This gives

$$
\begin{aligned}
& \sum_{\substack{z_{1} z_{2} \in E\left(Q_{\alpha}\left(H_{1}\right)\right) \\
z_{1} \in V\left(H_{1}\right) \\
z_{2} \in V\left(Q_{\alpha}\left(H_{1}\right)\right)-V\left(H_{1}\right)}}\left[d_{Q_{\alpha}\left(H_{1}\right)}\left(z_{2}\right)\right]=2 \sum_{u v \in E\left(H_{1}\right)}\left[d_{H_{1}}(u)+d_{H_{1}}(v)\right]=2 M_{1}\left(H_{1}\right) \\
& \sum_{\substack{H_{2} \\
z_{1} z_{2} \in E\left(Q_{\alpha}\left(H_{1}\right)\right) \\
z_{1} \in V\left(H_{1}\right) \\
z_{2} \in V\left(Q_{\alpha}\left(H_{1}\right)\right)-V\left(H_{1}\right)}}\left[d_{Q_{\alpha}\left(H_{1}\right)}\left(z_{2}\right)\right]=2\left|V\left(H_{2}\right)\right| M_{1}\left(H_{1}\right) .
\end{aligned}
$$

So, we have

$\sum_{B_{1}}=4\left|V\left(H_{2}\right)\left\|E\left(H_{1}\right)\right\| E\left(H_{2}\right)\right|+3\left|V\left(H_{2}\right)\right|^{3} M_{1}\left(H_{1}\right)$.
Now, we take

$$
\sum_{B_{2}}=\sum_{\substack{y \in V\left(H_{2}\right) \\ z_{1} z_{2} \in E\left(Q_{\alpha}\left(H_{1}\right)\right) \\ z_{1} z_{2} \in V\left(Q_{\alpha}\left(H_{1}\right)\right)-V\left(H_{1}\right)}}\left[d\left(z_{1}, y\right)+d\left(z_{2}, y\right)\right]
$$




$$
\begin{aligned}
& =\sum_{y \in V\left(H_{2}\right)} \sum_{\substack{z_{1} z_{2} \in E\left(Q_{\alpha}\left(H_{1}\right)\right) \\
z_{1} z_{2} \in V\left(Q_{\alpha}\left(H_{1}\right)\right)-V\left(H_{1}\right)}}\left[\left|V\left(H_{2}\right)\right| d_{Q_{\alpha}\left(H_{1}\right)}\left(z_{1}\right)+\left|V\left(H_{2}\right)\right| d_{Q_{\alpha}\left(H_{1}\right)}\left(z_{2}\right)\right] \\
& =2(\alpha-1)\left|V\left(H_{2}\right)\right|^{2} \sum_{u v \in E\left(H_{1}\right)}\left[d_{H_{1}}(u)+d_{H_{1}}(v)\right]=2(\alpha-1)\left|V\left(H_{2}\right)\right|{ }^{2} M_{1}\left(H_{1}\right), \\
& \sum_{B_{3}}=\sum_{y_{1} \in V\left(H_{2}\right)} \sum_{y_{2} \in V\left(H_{2}\right)} \sum_{z_{1} z_{2} \in E\left(Q_{\alpha}\left(H_{1}\right)\right)}\left[\left|V\left(\left(H_{2}\right)\right)\right| d_{Q_{\alpha}\left(H_{1}\right)}\left(z_{1}\right)+\left|V\left(H_{2}\right)\right| d_{Q_{\alpha}\left(H_{1}\right)}\left(z_{2}\right)\right] \\
& z_{1} z_{2} \in V\left(Q_{\alpha}\left(H_{1}\right)\right)-V\left(H_{1}\right) \\
& =\left|V\left(H_{2}\right)\right|^{3}(\alpha) \sum_{\substack{u v \in E\left(H_{1}\right) \\
v w \in E\left(H_{2}\right)}}\left[d_{H_{1}}(u)+d_{H_{1}}(v)+d_{H_{1}}(v)+d_{H_{1}}(w)\right] \text {, }
\end{aligned}
$$

where $z_{1}$ and $z_{2}$ are the vertices that are inserted into the edges $u v$ of $H_{1}$ and $v w$ of $H_{1}$, respectively.

$$
\begin{aligned}
& =(\alpha)\left|V\left(H_{2}\right)\right|^{3}\left[\sum_{v \in V\left(H_{1}\right)} d_{H_{1}}^{3}(v)-d_{H_{1}}^{2}(v)+\sum_{v \in V\left(H_{1}\right)}\left(d_{H_{1}}(v)-1\right) \sum_{\substack{u \in V\left(H_{1}\right) \\
u v \in E\left(H_{1}\right)}} d_{H_{1}}(u)\right] \\
& =(\alpha)\left|V\left(H_{2}\right)\right|^{3}\left[M_{3}\left(H_{1}\right)+2 M_{2}\left(H_{1}\right)-2 M_{1}\left(H_{1}\right)\right] .
\end{aligned}
$$

Consequently, we have obtained our required result.

Theorem 2. Let $H_{1}$ and $H_{2}$ be two connected graphs such that $\left|V\left(H_{1}\right)\right|,\left|V\left(H_{2}\right)\right| \geq 4$. For $\alpha \geq 1$,

$$
\begin{aligned}
M_{2}\left(H_{1_{(\alpha)}}\left[H_{2}\right]\right)= & 5\left|E ( H _ { 2 } ) \left\|\left.V\left(H_{2}\right)\right|^{2} M_{1}\left(H_{1}\right)+2\left|E\left(H_{1}\right) \| V\left(H_{2}\right)\right| M_{1}\left(H_{2}\right)+\left|V\left(H_{1}\right)\right| M_{2}\left(H_{2}\right)\right.\right. \\
& +\left|V\left(H_{2}\right)\right|^{4}\left[M_{3}\left(H_{1}\right)+2 M_{2}\left(H_{1}\right)\right]+(\alpha)\left|V\left(H_{2}\right)\right|^{4} \\
& {\left[\frac{1}{2} M_{4}\left(H_{1}\right)-\frac{1}{2} M_{3}\left(H_{1}\right)+\sum_{u v \in V\left(H_{1}\right)} r d(u) d(v)\right.} \\
& \left.+\sum_{v \in V\left(H_{1}\right)} d^{2}(v) \sum_{u \in V\left(H_{1}\right)} d(u)-2 M_{2}\left(H_{1}\right)\right]+(\alpha-1)\left|V\left(H_{2}\right)\right|^{3}\left[2 M_{2}\left(H_{1}\right)+M_{3}\left(H_{1}\right)\right], \\
&
\end{aligned}
$$


where $r$ is the number of neighbours which are common Proof. between $u$ and $v$ in $H_{1}$.

$$
\begin{aligned}
M_{2}\left(H_{1_{\left(Q_{\alpha}\right)}}\left[H_{2}\right]\right) & \left.=\sum_{\left(z_{1}, y_{1}\right)\left(z_{2}, y_{2}\right) \in E\left(H_{1}\left(Q_{\alpha}\right)\right.}\left[H_{2}\right]\right) \\
& =\sum_{z \in V\left(H_{1}\right)} \sum_{y_{1} y_{2} \in E\left(H_{2}\right)}\left[d\left(z, y_{1}\right) d\left(z, y_{2}\right)\right]+\sum_{y_{1} \in V\left(H_{2}\right)} \sum_{y_{2} \in V\left(H_{2}\right)} \sum_{y_{2} \in V\left(H_{2}\right)} \sum_{z_{1} z_{2} \in E\left(Q_{\alpha}\left(H_{1}\right)\right)}\left[d\left(z_{1}, y_{1}\right) d\left(z_{2}, y_{2}\right)\right] \\
& =\sum_{A}+\sum_{B} .
\end{aligned}
$$

Now, we take

$$
\begin{aligned}
& \sum_{A}=\sum_{z \in V\left(H_{1}\right)} \sum_{y_{1} y_{2} \in E\left(H_{2}\right)}\left[d\left(z, y_{1}\right) d\left(z, y_{2}\right)\right] \\
& =\sum_{z \in V\left(H_{1}\right)} \sum_{y_{1} y_{2} \in E\left(H_{2}\right)}\left[\left|V\left(H_{2}\right)\right| d_{H_{1}}(z)+d_{H_{2}}\left(y_{1}\right)\right]\left[\left|V\left(H_{2}\right)\right| d_{H_{1}}(z)+d_{H_{2}}\left(y_{2}\right)\right] \\
& =\left|E\left(H_{2}\right)\left\|\left.V\left(H_{2}\right)\right|^{2} M_{1}\left(H_{1}\right)+2\left|E\left(H_{1}\right) \| V\left(H_{2}\right)\right| M_{1}\left(H_{2}\right)+\left|V\left(H_{1}\right)\right| M_{2}\left(H_{2}\right)\right]\right. \text {, } \\
& \sum_{B}=\sum_{y_{1} \in V\left(H_{2}\right)} \sum_{y_{2} \in V\left(H_{2}\right)} \sum_{z_{1} z_{2} \in E\left(Q_{\alpha}\left(H_{1}\right)\right)}\left[d\left(z_{1}, y_{1}\right) d\left(z_{2}, y_{2}\right)\right] \\
& =\sum_{y_{1} \in V\left(H_{2}\right)} \sum_{y_{2} \in V\left(H_{2}\right)} \sum_{\substack{z_{1} z_{2} \in E\left(Q_{\alpha}\left(H_{1}\right)\right) \\
z_{1} \in V\left(H_{1}\right) \\
z_{2} \in V\left(Q_{\alpha}\left(H_{1}\right)\right)-V\left(H_{2}\right)}}\left[d\left(z_{1}, y_{1}\right) d\left(z_{2}, y_{2}\right)\right] \sum_{y \in V\left(H_{2}\right)} \sum_{\substack{z_{1} z_{2} \in E\left(Q_{\alpha}\left(H_{1}\right)\right) \\
z_{1} z_{2} \in V\left(Q_{\alpha}\left(H_{1}\right)\right)-V\left(H_{1}\right)}}\left[d\left(z_{1}, y\right) d\left(z_{2}, y\right)\right] \\
& +\sum_{y_{1} \in V\left(H_{2}\right)} \sum_{y_{2} \in V\left(H_{2}\right)} \sum_{\substack{z_{1} z_{2} \in E\left(Q_{\alpha}\left(H_{1}\right)\right) \\
z_{1} z_{2} \in V\left(Q_{\alpha}\left(H_{1}\right)\right)-V\left(H_{1}\right)}}\left[d\left(z_{1}, y_{1}\right) d\left(z_{2}, y_{2}\right)\right]=\sum_{B_{1}}+\sum_{B_{2}}+\sum_{B_{3}} .
\end{aligned}
$$

So,

$$
\begin{aligned}
\sum_{B_{1}} & =\sum_{y_{1} \in V\left(H_{2}\right)} \sum_{y_{2} \in V\left(H_{2}\right)} \sum_{\substack{z_{1} z_{2} \in E\left(Q_{\alpha}\left(H_{1}\right)\right) \\
z_{1} \in V\left(H_{1}\right) \\
z_{2} \in V\left(Q_{\alpha}\left(H_{1}\right)\right)-V\left(H_{1}\right)}}\left[d\left(z_{1}, y_{1}\right) d\left(z_{2}, y_{2}\right)\right] \\
& =\sum_{y_{1} \in V\left(H_{2}\right)} \sum_{y_{2} \in V\left(H_{2}\right)} \sum_{\substack{z_{1} z_{2} \in E\left(Q_{\alpha}\left(H_{1}\right)\right) \\
z_{1} \in V\left(H_{1}\right) \\
z_{2} \in V\left(Q_{\alpha}\left(H_{1}\right)\right)-V\left(H_{1}\right)}}\left[\| V\left(H_{2}\right)|| d_{H_{1}}\left(z_{1}\right)+d_{H_{2}}\left(y_{1}\right)\right]\left[\left|V\left(H_{2}\right)\right| d_{Q_{\alpha}\left(H_{1}\right)}\left(z_{2}\right)\right] \\
& \sum_{y_{1} \in V\left(H_{2}\right)} \sum_{y_{2} \in V\left(H_{2}\right)} \sum_{\begin{array}{l}
z_{1} z_{2} \in E\left(Q\left(H_{1}\right)\right) \\
z_{1} \in V\left(H_{1}\right) \\
z_{2} \in V\left(Q\left(H_{1}\right)\right)-V\left(H_{1}\right)
\end{array}}\left[\left|V\left(H_{2}\right)\right| d_{Q_{\alpha}\left(H_{1}\right)}\left(z_{2}\right) d_{\left(H_{2}\right)}\left(y_{1}\right)+\left|V\left(H_{2}\right)\right|^{2} d_{H_{1}}\left(z_{1}\right) d_{Q_{\alpha}\left(H_{1}\right)}\left(z_{2}\right)\right]
\end{aligned}
$$




$$
\begin{aligned}
& =\left|V\left(H_{2}\right)\right|^{4} \sum_{\substack{z_{1} z_{2} \in E\left(Q\left(H_{1}\right)\right) z_{1} \\
\epsilon V\left(H_{1}\right) z_{2} \in V\left(Q\left(H_{1}\right)\right)-V\left(H_{1}\right)}} d_{Q_{\alpha}\left(H_{1}\right)}\left(z_{1}\right) d_{Q_{\alpha}\left(H_{1}\right)}\left(z_{2}\right)+2\left|E\left(H_{2}\right) \| V\left(H_{2}\right)\right|^{2} \sum_{\substack{z_{1} z_{2} \in E\left(Q\left(H_{1}\right)\right) z_{1} \\
\epsilon V\left(H_{1}\right) \\
z_{2} \in V\left(Q\left(H_{1}\right)\right)-V\left(H_{1}\right)}} d_{Q_{\alpha}\left(H_{1}\right)}\left(z_{2}\right) \\
& =\left|V\left(H_{2}\right)\right|^{4} \sum_{\substack{z_{1} z_{2} \in E\left(Q\left(H_{1}\right)\right) z_{1} \in V \\
\left(H_{1}\right) z_{2} \in V\left(Q\left(H_{1}\right)\right)-V\left(H_{1}\right)}} d_{Q_{\alpha}\left(H_{1}\right)}\left(z_{1}\right) d_{Q_{\alpha}\left(H_{1}\right)}\left(z_{2}\right)+4\left|E\left(H_{2}\right) \| V\left(H_{2}\right)\right|^{2} M_{1}\left(H_{1}\right)
\end{aligned}
$$

where $z_{2}$ is the vertex inserted into the edge $z_{1} w_{1}$ of $H_{1}$

$$
\begin{aligned}
& =\left|V\left(H_{2}\right)\right|^{4} \sum_{z_{1} \in V\left(H_{1}\right)} \sum_{w_{1} \in V\left(N_{H_{1}}\left(z_{1}\right)\right)} d_{Q_{\alpha}\left(H_{1}\right)}\left(z_{1}\right)\left[d_{Q_{\alpha}\left(H_{1}\right)}\left(z_{1}\right)+d_{Q_{\alpha}\left(H_{1}\right)}\left(w_{1}\right)\right] \\
& +4\left|E\left(H_{2}\right) \| V\left(H_{2}\right)\right|^{2} M_{1}\left(H_{1}\right),
\end{aligned}
$$

where $N_{H_{1}}\left(z_{1}\right)$ is the set of neighbor vertices of $z_{1}$ in $H_{1}$

$$
\begin{aligned}
& =\left|V\left(H_{2}\right)\right|^{4}\left[M_{3}\left(H_{1}\right)+2 M_{2}\left(H_{1}\right)\right]+4\left|E\left(H_{2}\right) \| V\left(H_{2}\right)\right|^{2} M_{1}\left(H_{1}\right), \\
& \sum_{B_{2}}=\sum_{y \in V\left(H_{2}\right)} \sum_{z_{1} z_{2} \in E\left(Q_{\alpha}\left(H_{1}\right)\right)}\left[d\left(z_{1}, y\right) d\left(z_{2}, y\right)\right] \\
& z_{1} z_{2} \in V\left(Q_{\alpha}\left(H_{1}\right)\right)-V\left(H_{1}\right) \\
& \sum_{B_{2}}=\sum_{y \in V\left(H_{2}\right)} \sum_{z_{1} z_{2} \in E\left(Q_{\alpha}\left(H_{1}\right)\right)} \quad\left[\left|V\left(H_{2}\right)\right| d_{Q_{\alpha}\left(H_{1}\right)}\left(z_{1}\right)\right]\left[\left|V\left(H_{2}\right)\right| d_{Q_{\alpha}\left(H_{1}\right)}\left(z_{2}\right)\right] \\
& z_{1} z_{2} \in V\left(Q_{\alpha}\left(H_{1}\right)\right)-V\left(H_{1}\right) \\
& =(\alpha-1)\left|V\left(H_{2}\right)\right|^{3}\left[2 M_{2}\left(H_{1}\right)+M_{3}\left(H_{1}\right)\right], \\
& \sum_{B_{3}}=\sum_{y_{1} \in V\left(H_{2}\right)} \sum_{y_{2} \in V\left(H_{2}\right)} \sum_{z_{1} z_{2} \in E\left(Q_{\alpha}\left(H_{1}\right)\right)} \quad\left[\left|V\left(H_{2}\right)\right| d_{Q_{\alpha}\left(H_{1}\right)}\left(z_{1}\right)\right]\left[\left|V\left(H_{2}\right)\right| d_{Q_{\alpha}\left(H_{1}\right)}\left(z_{2}\right)\right] \\
& z_{1} z_{2} \in V\left(Q_{\alpha}\left(H_{1}\right)\right)-V\left(H_{1}\right) \\
& =\left|V\left(H_{2}\right)\right|^{2}(\alpha) \sum_{y_{1} \in V\left(H_{2}\right)} \sum_{y_{2} \in V\left(H_{2}\right)} \sum_{u v \in E\left(H_{1}\right)}\left[d_{H_{1}}(u)+d_{H_{1}}(v)\right]\left[d_{H_{1}}(v)+d_{H_{1}}(w)\right], \\
& v w \in E\left(H_{2}\right)
\end{aligned}
$$

where $z_{1}$ is the added vertex in the edge $u v$, and $z_{2}$ is the added vertex in the edges $v w$ of $H_{1}$ 


$$
\begin{aligned}
= & (\alpha)\left|V\left(H_{2}\right)\right|^{4}\left[\frac{1}{2} \sum_{v \in V\left(H_{1}\right)}\left(d_{H_{1}}^{4}(v)-d_{H_{1}}^{3}(v)\right)+\sum_{u v \in V\left(H_{1}\right)} r d_{H_{1}}(u) d_{H_{1}}(v)+\sum_{v \in V\left(H_{1}\right)} d_{H_{1}}^{2}(v)\right. \\
& \left.\times \sum_{\substack{u \in V\left(H_{1}\right) \\
u v \in E\left(H_{1}\right)}} d_{H_{1}}(u)-2 M_{2}\left(H_{1}\right)\right] \\
= & (\alpha)\left|V\left(H_{2}\right)\right|^{4}\left[\frac{1}{2} M_{4}\left(H_{1}\right)-\frac{1}{2} M_{3}\left(H_{1}\right)+\sum_{u v \in V\left(H_{1}\right)} r d_{H_{1}}(u) d_{H_{1}}(v)\right. \\
& \left.+\sum_{v \in V\left(H_{1}\right)} d_{H_{1}}^{2}(v) \sum_{\substack{u \in V\left(H_{1}\right) \\
u v \in E\left(\left(H_{1}\right)\right.}} d_{H_{1}}(u)-2 M_{2}\left(H_{1}\right)\right] .
\end{aligned}
$$

where $r$ is the number of neighbors which are common vertices of $u$ and $v$ in $\left(H_{1}\right)$. Consequently, we have obtained our required result.
Theorem 3. Let $H_{1}$ and $H_{2}$ be two connected graphs such that $\left|V\left(H_{1}\right)\right|,\left|V\left(H_{2}\right)\right| \geq 4$. For $\alpha \geq 1$,

$$
\begin{aligned}
F\left(H_{\left.1_{\left(Q_{\alpha}\right)}\left[H_{2}\right]\right)=}\right. & 3\left|V\left(H_{2}\right)\right|^{4} F\left(H_{1}\right)+\left|V\left(H_{1}\right)\right| F\left(H_{2}\right)+6\left|V\left(H_{2}\right)\right|^{2}\left|E\left(H_{2}\right)\right| M_{1}\left(H_{1}\right)+6\left|V\left(H_{2}\right)\right| \\
& \times\left|E\left(H_{1}\right)\right| M_{1}\left(H_{2}\right)+4\left|V\left(H_{2}\right)\right|^{4} M_{2}\left(H_{1}\right)+(\alpha)\left|V\left(H_{2}\right)\right|^{4}\left[M_{4}\left(H_{1}\right)-F\left(H_{1}\right)-4 M_{2}\left(H_{1}\right)\right. \\
& +\sum_{u \in V\left(H_{1}\right)}\left(\sum_{v \in N_{H_{1}}(u)} d_{H_{1}}(u)\left(d_{H_{1}}(v)-1\right)\right)+\sum_{u v \in E\left(H_{1}\right)} d_{H_{1}}(u) d_{H_{1}}(v)\left(d_{H_{1}}(u)\right. \\
& \left.\left.+d_{H_{1}}(v)\right)\right]+2(\alpha-1)\left|V\left(H_{2}\right)\right|^{3} F\left(H_{1}\right) .
\end{aligned}
$$

Proof.

Consider

$$
\begin{aligned}
F\left(H_{\left.1_{\left(Q_{\alpha}\right)}\left[H_{2}\right]\right)}=\right. & \left.\sum_{\left(z_{1}, y_{1}\right)\left(z_{2}, y_{2}\right) \in E\left(H_{1}\left(Q_{\alpha}\right)\right.}\left[H_{2}\right]\right) \\
& =\sum_{z \in V\left(H_{1}\right)} \sum_{y_{1} y_{2} \in E\left(H_{2}\right)}\left[d\left(z, y_{1}\right)^{2}+d\left(z, y_{2}\right)^{2}\right]+\sum_{y \in V\left(H_{2}\right)} \sum_{z_{1} z_{2} \in E\left(Q_{\alpha}\left(H_{2}\right)\right)}\left[d\left(z_{1}, y\right)^{2}+d\left(z_{2}, y\right)^{2}\right] \\
& =\sum_{A}+\sum_{B} .
\end{aligned}
$$

Now,

$$
\begin{aligned}
\sum_{A} & =\sum_{z \in V\left(H_{1}\right)} \sum_{y_{1} y_{2} \in E\left(H_{2}\right)}\left[\left(d_{H_{1}}(z)+d_{H_{2}}\left(y_{1}\right)\right)^{2}+\left(d_{H_{1}}(z)+d_{H_{2}}\left(y_{2}\right)\right)^{2}\right] \\
& =\sum_{z \in V\left(H_{1}\right)} \sum_{y_{1} y_{2} \in E\left(H_{2}\right)}\left[2\left|V\left(H_{2}\right)\right|^{2} d_{H_{1}}(z)^{2}+\left(d_{H_{2}}\left(y_{1}\right)^{2}+d_{H_{2}}\left(y_{2}\right)^{2}\right)+2 d_{H_{1}}(z)\left|V\left(H_{1}\right)\right|\left(d_{H_{2}}\left(y_{1}\right)+d_{H_{2}}\left(y_{2}\right)\right)\right] \\
& =\sum_{z \in V\left(H_{1}\right)}\left[\left|E\left(H_{2}\right) \| V\left(H_{2}\right)\right|^{2} \times 2 d_{H_{1}}(z)^{2}+F\left(H_{2}\right)+2\left|V\left(H_{2}\right)\right| M_{1}\left(H_{2}\right) d_{H_{1}}(z)\right]
\end{aligned}
$$




$$
\begin{aligned}
& =2\left|E ( H _ { 2 } ) \left\|\left.V\left(H_{2}\right)\right|^{2} M_{1}\left(H_{1}\right)+\left|V\left(H_{1}\right)\right| F\left(H_{2}\right)+4\left|V\left(H_{2}\right) \| E\left(H_{1}\right)\right| M_{1}\left(H_{2}\right),\right.\right. \\
& \sum_{B}=\sum_{y_{1} \in V\left(H_{2}\right)} \sum_{y_{2} \in V\left(H_{2}\right)} \sum_{z_{1} z_{2} \in E\left(Q_{\alpha}\left(H_{1}\right)\right)}\left[d\left(z_{1}, y_{1}\right)^{2}+d\left(z_{2}, y_{2}\right)^{2}\right] \\
& =\sum_{y_{1} \in V\left(H_{2}\right)} \sum_{\substack{y_{2} \in V\left(H_{2}\right) \\
z_{1} z_{2} \in E\left(Q_{\alpha}\left(H_{1}\right)\right) \\
z_{1} \in V\left(H_{1}\right) z_{2} \in V\left(Q_{\alpha}\left(H_{1}\right)\right)-V\left(H_{1}\right)}}\left[d\left(z_{1}, y_{1}\right)^{2}+d\left(z_{2}, y_{2}\right)^{2}\right] \\
& +\sum_{y \in V\left(H_{2}\right)} \sum_{\substack{z_{1} z_{2} \in E\left(Q_{k}\left(H_{1}\right)\right) \\
z_{1} z_{2} \in V\left(Q_{\alpha}\left(H_{1}\right)\right)-V\left(H_{1}\right)}}\left[d\left(z_{1}, y\right)^{2}+d\left(z_{2}, y\right)^{2}\right] \\
& +\sum_{y_{1} \in V\left(H_{2}\right)} \sum_{y_{2} \in V\left(H_{2}\right)} \sum_{\substack{z_{1} z_{2} \in E\left(Q_{\alpha}\left(H_{1}\right)\right) \\
z_{1} z_{2} \in V\left(Q_{\alpha}\left(H_{1}\right)\right)-V\left(H_{1}\right)}}\left[d\left(z_{1}, y_{1}\right)^{2}+d\left(z_{2}, y_{2}\right)^{2}\right]=\sum_{B_{1}}+\sum_{B_{2}}+\sum_{B_{3}} .
\end{aligned}
$$

Now, we take

$$
\begin{aligned}
& \sum_{B_{1}}=\sum_{y_{1} \in V\left(H_{2}\right)} \sum_{y_{2} \in V\left(H_{2}\right)} \sum_{\substack{z_{1} z_{2} \in E\left(Q_{\alpha}\left(H_{1}\right)\right) \\
z_{1} \in V\left(H_{1}\right) z_{2} \in V\left(Q_{\alpha}\left(H_{1}\right)\right)-V\left(H_{1}\right)}}\left[\left(\left|V\left(H_{2}\right)\right| d_{Q_{\alpha}\left(H_{1}\right)}\left(z_{1}\right)+d_{H_{2}}\left(y_{1}\right)\right)^{2}+\left|V\left(H_{2}\right)\right|^{2} d_{Q_{\alpha}\left(H_{1}\right)}\left(z_{2}\right)^{2}\right]
\end{aligned}
$$

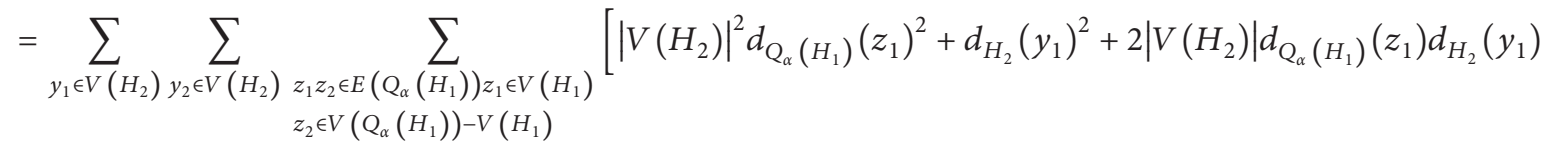

$$
\begin{aligned}
& \left.+\left|V\left(H_{2}\right)\right|^{2} d_{Q_{\alpha}\left(H_{1}\right)}\left(z_{2}\right)^{2}\right] \\
& =\sum_{y_{1} \in V\left(H_{2}\right)} \sum_{y_{2} \in V\left(H_{2}\right)} \sum_{z_{1} \in V\left(H_{1}\right)}\left[\left|V\left(H_{2}\right)\right|^{2} d_{Q_{\alpha}\left(H_{1}\right)}\left(z_{1}\right)^{2}+d_{H_{2}}\left(y_{1}\right)^{2}+2\left|V\left(H_{2}\right)\right| d_{Q_{\alpha}\left(H_{1}\right)}\left(z_{1}\right) d_{H_{2}}\left(y_{1}\right)\right] \\
& +\sum_{y_{1} \in V\left(H_{2}\right)} \sum_{y_{2} \in V\left(H_{2}\right)} \sum_{z_{1} z_{2} \in E\left(Q_{\alpha}\left(H_{1}\right)\right) z_{1} \in V\left(H_{1}\right)}\left[\left|V\left(H_{2}\right)\right|^{2} d_{Q_{\alpha}\left(H_{1}\right)}\left(z_{2}\right)^{2}\right] \\
& z_{2} \in V\left(Q_{\alpha}\left(H_{1}\right)\right)-V\left(H_{1}\right) \\
& =\left|V\left(H_{2}\right)\right| F\left(H_{1}\right)+2\left|E\left(H_{1}\right)\right| M_{1}\left(H_{2}\right)+4\left|E\left(H_{2}\right)\right| M_{1}\left(H_{1}\right)+\left|V\left(H_{2}\right)\right|^{2} \sum_{y \in V\left(H_{2}\right)} \\
& \times \sum_{\substack{z_{1} z_{2} \in E\left(Q_{\alpha}\left(H_{1}\right)\right) z_{1} \in V\left(H_{1}\right) \\
z_{2} \in V\left(Q_{\alpha}\left(H_{1}\right)\right)-V\left(H_{1}\right)}}\left[d_{Q_{\alpha}\left(H_{1}\right)}\left(z_{2}\right)^{2}\right] .
\end{aligned}
$$

For $z_{2} \in V\left(Q_{\alpha}\left(H_{1}\right)\right)-V\left(H_{1}\right)$ that is introduced into the edges $u v$ of $\left(H_{1}\right)$, we have $d_{Q_{\alpha}\left(H_{1}\right)}\left(z_{2}\right)=d_{H_{1}}(u)+d_{H_{1}}(v)$. This gives

$$
\sum_{z_{1} z_{2} \in E\left(Q_{\alpha}\left(H_{1}\right)\right) z_{1} \in V\left(H_{1}\right)}\left[d_{Q_{\alpha}\left(H_{1}\right)}\left(z_{2}\right)^{2}\right]=2 \sum_{u v \in E\left(H_{1}\right)}\left[d_{H_{1}}(u)+d_{H_{1}}(v)\right]^{2}=2 H M\left(H_{1}\right) .
$$

So, 


$$
\begin{aligned}
& \sum_{B_{1}}=\left|V\left(H_{2}\right)\right|^{4}\left[2 H M\left(H_{1}\right)+F\left(H_{1}\right)\right]+2\left|V\left(H_{2}\right) \| E\left(H_{1}\right)\right| M_{1}\left(H_{2}\right)+4\left|V\left(H_{2}\right)\right|^{2}\left|E\left(H_{2}\right)\right| M_{1}\left(H_{1}\right), \\
& \sum_{B_{2}}=\sum_{y \in V\left(H_{2}\right)} \sum_{z_{1} z_{2} \in E\left(Q_{\alpha}\left(H_{1}\right)\right)} \quad\left[\left|V\left(H_{2}\right)\right|^{2} d\left(z_{1}, y\right)^{2}+\left|V\left(H_{2}\right)\right|^{2} d\left(z_{2}, y\right)^{2}\right] \\
& z_{1}, z_{2} \in V\left(Q_{\alpha}\left(H_{1}\right)\right)-V\left(H_{1}\right) \\
& =\sum_{y \in V\left(H_{2}\right)} \sum_{z_{1} z_{2} \in E\left(Q_{\alpha}\left(H_{1}\right)\right)} \quad\left[\left|V\left(H_{2}\right)\right|^{2} d_{Q_{\alpha}\left(H_{1}\right)}\left(z_{1}\right)^{2}+\left|V\left(H_{2}\right)\right|^{2} d_{Q_{\alpha}\left(H_{1}\right)}\left(z_{2}\right)^{2}\right] \\
& z_{1} z_{2} \in V\left(Q_{\alpha}\left(H_{1}\right)\right)-V\left(H_{1}\right) \\
& =2(\alpha-1)\left|V\left(H_{2}\right)\right|^{2} \sum_{y \in V\left(H_{2}\right)} \sum_{u v \in E\left(H_{1}\right)}\left[d_{H_{1}}(u)^{2}+d_{H_{1}}(v)^{2}\right]=2(\alpha-1)\left|V\left(H_{2}\right)\right|^{3} F\left(H_{1}\right), \\
& \sum_{B_{3}}=\sum_{y_{1} \in V\left(H_{2}\right)} \sum_{y_{2} \in V\left(H_{2}\right)} \sum_{z_{1} z_{2} \in E\left(Q_{\alpha}\left(H_{1}\right)\right)} \quad\left[\left|V\left(H_{2}\right)\right|^{2} d_{Q_{\alpha}\left(H_{1}\right)}\left(z_{1}\right)^{2}+\left|V\left(H_{2}\right)\right|^{2} d_{Q_{\alpha}\left(H_{1}\right)}\left(z_{2}\right)^{2}\right] \\
& z_{1}, z_{2} \in V\left(Q_{\alpha}\left(H_{1}\right)\right)-V\left(H_{1}\right) \\
& =(\alpha)\left|V\left(H_{2}\right)\right|^{4} \sum_{\substack{u v \in E\left(H_{1}\right) \\
v w \in E\left(H_{2}\right)}}\left[\left(d_{H_{1}}(u)+d_{H_{1}}(v)\right)^{2}+\left(d_{H_{1}}(v)+d_{H_{1}}(w)^{2}\right],\right.
\end{aligned}
$$

where $z_{1}$ and $z_{2}$ are the nodes that are introduced into the edge set of $u v$ and $v w$ of $H_{1}$

$$
\begin{aligned}
= & (\alpha)\left|V\left(H_{2}\right)\right|^{4}\left[\sum_{u \in V\left(H_{1}\right)} d_{H_{1}}(v)^{4}-d_{H_{1}}(v)^{3}+\sum_{v \in V\left(H_{1}\right)}\left(d_{H_{1}}(v)-1\right) \sum_{\substack{u \in V\left(H_{1}\right) \\
u v \in E\left(H_{1}\right)}} d_{H_{1}}(u)^{2}\right. \\
& \left.+2 \sum_{v \in V\left(H_{1}\right)} d_{H_{1}}(v)\left(d_{H_{1}}(v)-1\right) \sum_{\substack{u \in V\left(H_{1}\right) \\
u v \in E\left(H_{1}\right)}} d_{H_{1}}(u)\right] \\
= & (\alpha)\left|V\left(H_{2}\right)\right|^{4}\left[M_{4}\left(H_{1}\right)-F\left(H_{1}\right)-4 M_{2}\left(H_{1}\right)+\sum_{u \in V\left(H_{1}\right)}\left(\sum_{v \in N_{H_{1}}(u)} d_{H_{1}}(u)\left(d_{H_{1}}(v)-1\right)\right)\right. \\
& \left.+\sum_{u v \in E\left(H_{1}\right)} d_{H_{1}}(u) d_{H_{1}}(v)\left(d_{H_{1}}(u)+d_{H_{1}}(v)\right)\right],
\end{aligned}
$$

Consequently, we have obtained our required result.

Theorem 4. Let $H 1$ and $H 2$ be two connected graphs such that $|V(H 1)|,|V(H 2)| \geq 4$. For $\alpha \geq 1$,

$$
\begin{aligned}
H Z\left(H_{1_{\left(Q_{\alpha}\right)}}\left[H_{2}\right]\right)= & \alpha\left|V\left(H_{2}\right)\right|^{4}\left[H Z\left(L\left(H_{1}\right)\right)+8 M_{1}\left(L\left(H_{1}\right)+8 M_{1}\left(H_{1}\right)-16\left|E\left(H_{1}\right)\right|\right]\right. \\
& +4(\alpha-1)\left|V\left(H_{2}\right)\right|^{3} H Z\left(H_{1}\right)+12\left|V\left(H_{2}\right)\right|^{2}\left|E\left(H_{2}\right)\right| M_{1}\left(H_{1}\right)+\left|V\left(H_{1}\right)\right| H Z\left(H_{2}\right) \\
& +10|| V\left(H_{2}\right) \|\left. E\left(H_{1}\right)\left|M_{1}\left(H_{2}\right)+\right| V\left(H_{2}\right)\right|^{4}\left[2 H Z\left(H_{1}\right)+3 F\left(H_{1}\right)+4 M_{2}\left(H_{1}\right)\right] .
\end{aligned}
$$


Proof.

$$
\begin{aligned}
H Z\left(H_{1_{\left(Q_{\alpha}\right)}}\left[H_{2}\right]\right)= & \left.\sum_{\left(z_{1}, y_{1}\right)\left(z_{2}, y_{2}\right) \in E\left(H_{1}\left(Q_{\alpha}\right)\right.}\left[H_{2}\right]\right) \\
& \left.\left.\sum_{z \in V\left(H_{1}\right)} \sum_{y_{1} y_{2} \in E\left(H_{2}\right)}\left[d\left(z, y_{1}\right)+d\left(z, y_{2}\right)\right]^{2}+\sum_{y_{1} \in V\left(H_{2}\right)} \sum_{y_{2} \in V\left(H_{2}\right)} \sum_{z_{1} z_{2} \in E\left(Q_{\alpha}\left(H_{1}\right)\right)}\left[d\left(z_{2}, y_{2}\right)\right]^{2}, y_{1}\right)+d\left(z_{2}, y_{2}\right)\right]^{2} \\
= & \sum_{A}+\sum_{B} .
\end{aligned}
$$

Now,

$$
\begin{aligned}
& \sum_{A}=\sum_{z \in V\left(H_{1}\right)} \sum_{y_{1} y_{2} \in E\left(H_{2}\right)}\left[d\left(z, y_{1}\right)+d\left(z, y_{2}\right)\right]^{2} \\
& =\sum_{z \in V\left(H_{1}\right)} \sum_{y_{1} y_{2} \in E\left(H_{2}\right)}\left[\left(\left|V\left(H_{2}\right)\right| d_{Q_{\alpha}\left(H_{1}\right)}(z)+d_{H_{2}}\left(y_{1}\right)\right)+\left(\left|V\left(H_{2}\right)\right| d_{Q_{\alpha}\left(H_{1}\right)}(z)+d_{H_{2}}\left(y_{2}\right)\right)\right]^{2} \\
& =\sum_{z \in V\left(H_{1}\right)} \sum_{y_{1} y_{2} \in E\left(H_{2}\right)}\left[2\left|V\left(H_{2}\right)\right| d_{Q_{\alpha}\left(H_{1}\right)}(z)+d_{H_{2}}\left(y_{1}\right)+d_{H_{2}}\left(y_{2}\right)\right]^{2} \\
& =\sum_{z \in V\left(H_{1}\right)} \sum_{y_{1} y_{2} \in E\left(H_{2}\right)}\left[4\left|V\left(H_{2}\right)\right|^{2}\left(d_{Q_{\alpha}\left(H_{1}\right)}(z)\right)^{2}+\left(d_{H_{2}}\left(y_{1}\right)\right)^{2}+\left(d_{H_{2}}\left(y_{2}\right)\right)^{2}+4\left|V\left(H_{2}\right)\right| d_{Q_{\alpha}\left(H_{1}\right)}(z) d_{H_{2}}\left(y_{1}\right)\right. \\
& \left.+4\left|V\left(H_{2}\right)\right| d_{Q_{\alpha}\left(H_{1}\right)}(z) d_{H_{2}}\left(y_{2}\right)+2 d_{H_{2}}\left(y_{1}\right) d_{H_{2}}\left(y_{2}\right)\right] \\
& =4\left|E\left(H_{2}\right) \| V\left(H_{2}\right)\right|^{2} M_{1}\left(H_{1}\right)+\left|V\left(H_{1}\right)\right| H Z\left(H_{2}\right)+\sum_{z \in V\left(H_{1}\right)} \sum_{y_{1} y_{2} \in E\left(H_{2}\right)} 4 d_{H_{1}}(z)\left[d_{H_{2}}\left(y_{1}\right)+d_{H_{2}}\left(y_{2}\right)\right] \\
& =4\left|E ( H _ { 2 } ) \left\|\left.V\left(H_{2}\right)\right|^{2} M_{1}\left(H_{1}\right)+\left|V\left(H_{1}\right)\right| H Z\left(H_{2}\right)+8\left|E\left(H_{1}\right) \| V\left(H_{2}\right)\right| M_{1}\left(H_{2}\right),\right.\right. \\
& \sum_{B}=\sum_{y_{1} \in V\left(H_{2}\right)} \sum_{y_{2} \in V\left(H_{2}\right)} \sum_{z_{1} z_{2} \in E\left(Q_{\alpha}\left(H_{1}\right)\right)}\left[d\left(z_{1}, y\right)+d\left(z_{2}, y\right)\right]^{2} \\
& =\sum_{y_{1} \in V\left(H_{2}\right)} \sum_{y_{2} \in V\left(H_{2}\right)} \sum_{\substack{z_{1} z_{2} \in E\left(Q_{\alpha}\left(H_{1}\right)\right) \\
z_{1} \in V\left(H_{1}\right) z_{2} \in V\left(Q_{\alpha}\left(H_{1}\right)\right)-V\left(H_{1}\right)}}\left[d\left(z_{1}, y_{1}\right)+d\left(z_{2}, y_{2}\right)\right]^{2} \\
& +\sum_{y \in V\left(H_{2}\right)} \sum_{z_{1} z_{2} \in E\left(Q_{\alpha}\left(H_{1}\right)\right)}\left[d\left(z_{1}, y\right)+d\left(z_{2}, y\right)\right]^{2} \\
& z_{1} z_{2} \in V\left(Q_{\alpha}\left(H_{1}\right)\right)-V\left(H_{1}\right) \\
& +\sum_{y_{1} \in V\left(H_{2}\right)} \sum_{y_{2} \in V\left(H_{2}\right)} \sum_{z_{1} z_{2} \in E\left(Q_{\alpha}\left(H_{1}\right)\right)}\left[d\left(z_{1}, y_{1}\right)+d\left(z_{2}, y_{2}\right)\right]^{2}=\sum_{B_{1}}+\sum_{B_{2}}+\sum_{B_{3}} \text {. } \\
& z_{1} z_{2} \in V\left(Q_{\alpha}\left(H_{1}\right)\right)-V\left(H_{1}\right)
\end{aligned}
$$


Now, we take

$$
\begin{aligned}
& \sum_{B_{1}}=\sum_{y_{1} \in V\left(H_{2}\right)} \sum_{\substack{y_{2} \in V\left(H_{2}\right) \\
z_{1} z_{2} \in E\left(Q_{\alpha}\left(H_{1}\right)\right) \\
z_{1} \in V\left(H_{1}\right) z_{2} \in V\left(Q_{\alpha}\left(H_{1}\right)\right)-V\left(H_{1}\right)}}\left[d\left(z_{1}, y_{1}\right)+d\left(z_{2}, y_{2}\right)\right]^{2} \\
& =\sum_{y_{1} \in V\left(H_{2}\right)} \sum_{y_{2} \in V\left(H_{2}\right)} \sum_{\substack{z_{1} z_{2} \in E\left(Q_{\alpha}\left(H_{1}\right)\right) \\
z_{1} \in V\left(H_{1}\right) z_{2} \in V\left(Q_{\alpha}\left(H_{1}\right)\right)-V\left(H_{1}\right)}}\left[\left|V\left(H_{2}\right)\right| d_{Q_{\alpha}\left(H_{1}\right)}\left(z_{1}\right)+d_{H_{2}}\left(y_{1}\right)+\left|V\left(H_{2}\right)\right| d_{Q_{\alpha}\left(H_{1}\right)}\left(z_{2}\right)\right]^{2} \\
& =\sum_{y_{1} \in V\left(H_{2}\right)} \sum_{\substack{y_{2} \in V\left(H_{2}\right) \\
z_{1} z_{2} \in E\left(Q_{\alpha}\left(H_{1}\right)\right) \\
z_{1} \in V\left(H_{1}\right) z_{2} \in V\left(Q_{\alpha}\left(H_{1}\right)\right)-V\left(H_{1}\right)}}\left[\left|V\left(H_{2}\right)\right|^{2} d_{Q_{\alpha}\left(H_{1}\right)}\left(z_{1}\right)^{2}+d_{H_{2}}\left(y_{1}\right)^{2}+\left|V\left(H_{2}\right)\right|^{2} d_{Q_{\alpha}\left(H_{1}\right)}\left(z_{2}\right)^{2}\right. \\
& \left.+2\left|V\left(H_{2}\right)\right| d_{Q_{\alpha}\left(H_{1}\right)}\left(z_{1}\right) d_{H_{2}}\left(y_{1}\right)+2\left|V\left(H_{2}\right)\right| d_{H_{2}}\left(y_{1}\right) d_{Q_{\alpha}\left(H_{1}\right)}\left(z_{2}\right)+2\left|V\left(H_{2}\right)\right|^{2} d_{Q_{\alpha}\left(H_{1}\right)}\left(z_{1}\right) d_{Q_{\alpha}\left(H_{1}\right)}\left(z_{2}\right)\right] \\
& =\left|V\left(H_{2}\right)\right|^{4} F\left(H_{1}\right)+2\left|V\left(H_{2}\right) \| E\left(H_{1}\right)\right| M_{1}\left(H_{2}\right)+4\left|V\left(H_{2}\right)\right|^{2}\left|E\left(H_{2}\right)\right| M_{1}\left(H_{1}\right)+\sum_{y_{1} \in V\left(H_{2}\right)} \sum_{y_{2} \in V\left(H_{2}\right)} \\
& \times \sum_{\substack{z_{1} z_{\epsilon} \in E\left(Q_{\alpha}\left(H_{1}\right)\right) z_{1} \in V\left(H_{1}\right) \\
z_{2} \in V\left(Q_{\alpha}\left(H_{1}\right)\right)-V\left(H_{1}\right)}}\left[\left|V\left(H_{2}\right)\right|^{2} d_{Q_{\alpha}\left(H_{1}\right)}\left(z_{2}\right)^{2}+\left(2\left|V\left(H_{2}\right)\right|^{2} d_{H_{1}}\left(z_{1}\right)+2\left|V\left(H_{2}\right)\right|^{2} d_{Q_{\alpha}\left(H_{1}\right)}\left(z_{1}\right)\right) d_{Q_{\alpha}\left(H_{1}\right)}\left(z_{2}\right)\right] .
\end{aligned}
$$

One can see that for a vertex $z_{2} \in V\left(Q_{\alpha}\left(H_{1}\right)\right)-V\left(H_{1}\right)$, $d_{Q_{\alpha}\left(H_{1}\right)}\left(z_{2}\right)=d_{H_{1}}(z)+d_{H_{1}}(w)$, where $z_{2}=x w \in E\left(H_{1}\right)$. Thus,

$$
\begin{aligned}
& =\left|V\left(H_{2}\right)\right|^{4} F\left(\left(H_{1}\right)\right)+2\left|V\left(H_{2}\right) \| E\left(H_{1}\right)\right| M_{1}\left(H_{2}\right)+4\left|V\left(H_{2}\right)\right|^{2}\left|E\left(H_{2}\right)\right| M_{1}\left(H_{1}\right)+\sum_{y_{1} \in V\left(H_{2}\right)} \sum_{y_{2} \in V\left(H_{2}\right)} \\
& \times \sum_{\substack{z_{1} z_{2} \in E\left(Q_{\alpha}\left(H_{1}\right)\right) z_{1} \\
\in V\left(H_{1}\right) z_{2} \in V\left(Q_{\alpha}\left(H_{1}\right)\right)-V\left(H_{1}\right)}}\left[\left|V\left(H_{2}\right)\right|^{2}\left(d_{H_{1}}(z)+d_{H_{1}}(w)\right)^{2}+2\left|V\left(H_{2}\right)\right|^{2}\left(d_{H_{1}}\left(z_{1}\right)+d_{Q_{\alpha}\left(H_{1}\right)}\left(z_{1}\right)\right)\left(d_{H_{1}}(z)+d_{H_{1}}(w)\right)\right] \\
& =\left|V\left(H_{2}\right)\right|^{4} F\left(H_{1}\right)+2\left|V\left(H_{2}\right)\right|\left|E\left(H_{1}\right)\right| M_{1}\left(H_{2}\right)+4\left|V\left(H_{2}\right)\right|^{2}\left|E\left(H_{2}\right)\right| M_{1}\left(H_{1}\right) \\
& +2\left|V\left(H_{2}\right)\right|^{4} H Z\left(H_{1}\right)+2\left|V\left(H_{2}\right)\right|^{4}\left(F\left(H_{1}\right)+2 M_{2}\left(H_{1}\right)\right)+8\left|V\left(H_{2}\right)\right|^{2}\left|E\left(H_{1}\right)\right| M_{1}\left(H_{2}\right) \\
& =2\left|V\left(H_{2}\right)\right|^{4} H Z\left(H_{1}\right)+3\left|V\left(H_{2}\right)\right|^{4} F\left(H_{1}\right)+12\left|V\left(H_{2}\right)\right|^{2}\left|E\left(H_{2}\right)\right| M_{1}\left(H_{1}\right) \\
& +2\left|V\left(H_{2}\right) \| E\left(H_{1}\right)\right| M_{1}\left(H_{2}\right)+4\left|V\left(H_{2}\right)\right|^{4} M_{2}\left(H_{1}\right), \\
& \sum_{B_{2}}=\sum_{\substack{y \in V\left(H_{2}\right) \\
z_{1} z_{2} \in E\left(Q_{\alpha}\left(H_{1}\right)\right) \\
z_{1}, z_{2} \in V\left(Q_{\alpha}\left(H_{1}\right)\right)-V\left(H_{1}\right)}}\left[\left|V\left(H_{2}\right)\right| d_{Q_{\alpha}\left(H_{1}\right)}\left(z_{1}\right)+\left|V\left(H_{2}\right)\right| d_{Q_{\alpha}\left(H_{1}\right)}\left(z_{2}\right)\right]^{2} \\
& =4(\alpha-1)\left|V\left(H_{2}\right)\right|^{2} \sum_{y \in V\left(H_{2}\right)} \sum_{u v \in E\left(H_{1}\right)}\left[d_{H_{1}}(u)+d_{H_{1}}(v)\right]^{2}=4(\alpha-1)\left|V\left(H_{2}\right)\right|^{3} H M\left(H_{1}\right), \\
& \sum_{B_{3}}=\sum_{y_{1} \in V\left(H_{2}\right)} \sum_{y_{2} \in V\left(H_{2}\right)} \sum_{\substack{z_{1} z_{2} \in E\left(Q_{\alpha}\left(H_{1}\right)\right) \\
z_{1} z_{2} \in V\left(Q_{\alpha}\left(H_{1}\right)\right)-V\left(H_{1}\right)}}\left[\left|V\left(H_{2}\right)\right| d_{\mathrm{Q}_{\alpha}\left(H_{1}\right)}\left(z_{1}\right)+\left|V\left(H_{2}\right)\right| d_{\mathrm{Q}_{\alpha}\left(H_{1}\right)}\left(z_{2}\right)\right]^{2} \\
& =\alpha\left|V\left(H_{2}\right)\right|^{4} \sum_{\substack{u v \in\left(H_{1}\right) \\
v w \in E\left(H_{2}\right)}}\left[d_{H_{1}}(u)+d_{H_{1}}(v)+d_{H_{1}}(v)+d_{H_{1}}(w)\right]^{2} .
\end{aligned}
$$


TABle 1: $P_{n}\left[P_{m}\right]$.

\begin{tabular}{lcccccc}
\hline$[m, n]$ & $V$ & $\mathrm{E}$ & $M_{1}$ & $M_{2}$ & $F(H)$ & $H(H)$ \\
\hline$(3,3)$ & 9 & 24 & 276 & 764 & 3868 & 1704 \\
$(4,4)$ & 16 & 60 & 968 & 12576 & 280 & 26460 \\
$(5,5)$ & 25 & 120 & 2460 & 32132 & 66948 & 51612 \\
$(6,6)$ & 36 & 210 & 9196 & 70276 & 145488 & 131212 \\
$(7,7)$ & 49 & 336 & 9716 & 286040 \\
\hline
\end{tabular}

TABLe 2: $P_{n_{\left(Q_{\alpha}\right)}}\left[P_{m}\right]$.

\begin{tabular}{lcccccc}
\hline$[m, n, k]$ & $V$ & $\mathrm{E}$ & $M_{1}$ & $M_{2}$ & $F(H)$ & $H(H)$ \\
\hline$(3,3,3)$ & 27 & 189 & 6366 & 53701 & 114786 & 222188 \\
$(4,4,4)$ & 64 & 944 & 69024 & 1256160 & 2745760 & 5258080 \\
$(5,5,5)$ & 125 & 3175 & 392030 & $1.2195692 \times 10^{7}$ & $2.581541 \times 10^{7}$ & $5.0206794 \times 10^{7}$ \\
$(6,6,6)$ & 216 & 8424 & 1561992 & $7.3089364 \times 10^{7}$ & $1.52239968 \times 10^{8}$ & $2.98418696 \times 10^{8}$ \\
$(7,7,7)$ & 343 & 19061 & 4939998 & $3.22992564 \times 10^{8}$ & $6.66343762 \times 10^{8}$ & $1.31232889 \times 10^{9}$ \\
\hline
\end{tabular}

TABle 3: Correlation coefficient between $P_{n_{\left(Q_{\alpha}\right)}}\left[P_{m}\right]$ and $P_{n}\left[P_{m}\right]$.

\begin{tabular}{lcc}
\hline TI's & Value of $R$ & Relationship between $X$ and $Y$ \\
\hline First Zagreb index & 0.9756 & Very strong direct relationship \\
Second Zagreb index & 0.953 & Very strong direct relationship \\
Forgotten Zagreb index & 0.9123 & Very strong direct relationship \\
Hyper Zagreb index & 0.9736 & Very strong direct relationship \\
\hline
\end{tabular}

TABLE 4: Square of correlation coefficient between $P_{n_{\left(Q_{\alpha}\right)}}\left[P_{m}\right]$ and $P_{n}\left[P_{m}\right]$.

\begin{tabular}{lcc}
\hline TI’s & Value of $R^{2}$ & Variability of $Y$ is explained by $X$ \\
\hline First Zagreb index & 0.9518 & 95.1 percent \\
Second Zagreb index & 0.9080 & 90.8 percent \\
Forgotten Zagreb index & 0.8323 & 83.23 percent \\
Hyper Zagreb index & 0.9478 & 94.78 percent \\
\hline
\end{tabular}

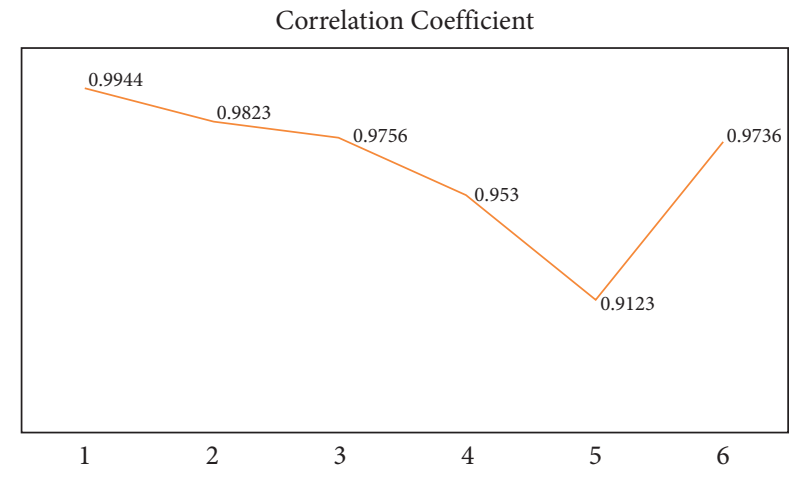

Figure 5: Value of $R$ for number of vertices, edges, first, second, forgotten, and hyper Zagreb indices.

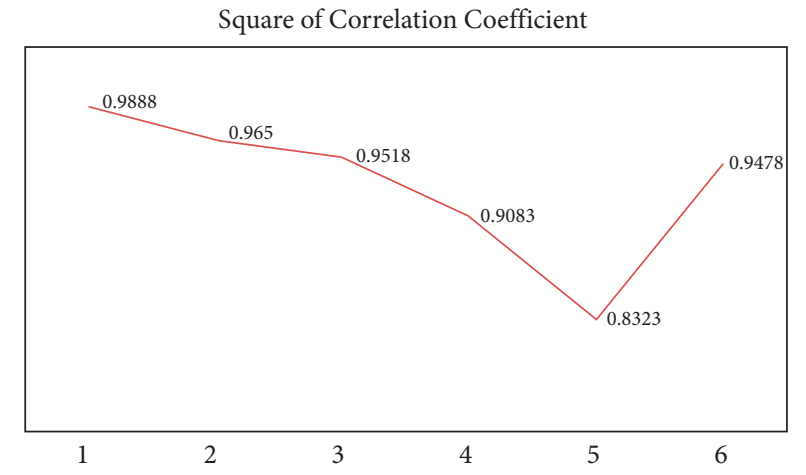

FIgURE 6: Value of $R^{2}$ for number of vertices, edges, first, second, forgotten, and hyper Zagreb indices. 
$W$ and $X$ are the nodes of $L\left(H_{1}\right)$, so we have

$$
\begin{aligned}
&= \alpha\left|V\left(H_{2}\right)\right|^{4} \sum_{W X \in E\left(L\left(H_{1}\right)\right)}\left[d_{L\left(H_{1}\right)}(W)^{2}+d_{L\left(H_{1}\right)}(X)^{2}\right. \\
&\left.+16+2 d_{L\left(H_{1}\right)}(W) d_{L\left(H_{1}\right)}(X)+8 d_{L\left(H_{1}\right)}(W)+8 d_{L\left(H_{1}\right)}(X)\right] \\
&= \alpha\left|V\left(H_{2}\right)\right|^{4}\left[H Z\left(L\left(H_{1}\right)\right)+8 M_{1}\left(L\left(H_{1}\right)\right)+16\left(\frac{1}{2} M_{1}\left(H_{1}\right)-E\left(H_{1}\right)\right)\right], \\
& \sum_{B_{3}}=\alpha\left|V\left(H_{2}\right)\right|^{4}\left[H Z\left(L\left(H_{1}\right)\right)+8 M_{1}\left(L\left(H_{1}\right)\right)+8 M_{1}\left(H_{1}\right)-16 E\left(H_{1}\right)\right] .
\end{aligned}
$$

Consequently, the result is over.

\section{Applications and Conclusion}

For any two simple path graphs $P_{n}$ and $P_{m}$ with $n, m>3$, we construct their lexicographic product graphs $P_{n}\left[P_{m}\right]$ and compute various degree-related TIs, as shown in Table 1 with certain values of $m$ and $n$. Furthermore, we construct $Q_{\alpha}\left(P_{n}\right)$ by applying generalized line superposition operation on $P_{n}$ and then construct $Q_{\alpha}$-sum graph $\left(P_{n_{\left(Q_{n}\right)}}\left[P_{m}\right]\right)$ based on lexicographic product of $Q_{\alpha}\left(P_{n}\right)$ and $P_{m}$. Finally, we compute certain degree-related TIs using Theorem 1 -Theorem 4, as given in Table 2 . We note that by the addition of vertices $\alpha \geq 1$, the values of obtained TIs are increasing for the newly constructed graphs comparatively to the graphs obtained by the ordinary lexicographic product of graphs.

3.1. Linear Regression Model. To check the correlation between the predefined lexicographic product of two simple graphs $\left(P_{n}\left[P_{m}\right]\right)$ and newly defined $Q_{\alpha}$-sum graphs $\left(P_{n_{\left(Q_{\alpha}\right)}}\left[P_{m}\right]\right)$, we have made the linear regression model for all the obtained indices as follows:

\subsection{First Zagreb Index.}

$$
M_{1}\left(P_{n}\left[P_{m}\right]\right)=b_{0}+b_{1}\left[M_{1}\left(P_{n_{\left(Q_{\alpha}\right)}}\left[P_{m}\right]\right)\right],
$$

where $Y=M_{1}\left(P_{n}\left[P_{m}\right]\right)$ index values, $b_{0}$ is the constant, $b_{1}$ is the regression coefficient, and $X=M_{1}\left(P_{n_{\left(Q_{\alpha}\right)}}\left[P_{m}\right]\right)$ index values (see Tables 1 and 2).

$$
Y=1207.7339+0.001805 X
$$

\subsection{Second Zagreb Index.}

$$
M_{2}\left(P_{n}\left[P_{m}\right]\right)=b_{0}+b_{1}\left[M_{2}\left(P_{n_{\left(Q_{\alpha}\right)}}\left[P_{m}\right]\right)\right]
$$

where $Y=M_{2}\left(P_{n}\left[P_{m}\right]\right)$ index values, $b_{0}$ is the constant, $b_{1}$ is the regression coefficient, and $X=M_{2}\left(P_{n_{\left(Q_{\alpha}\right)}}\left[P_{m}\right]\right)$ index values (see Tables 1 and 2).

$$
Y=1548.9242+0.00002654 X \text {. }
$$

\subsection{Forgotten Topological Index.}

$$
F\left(P_{n}\left[P_{m}\right]\right)=b_{0}+b_{1}\left[F\left(P_{n_{\left(Q_{\alpha}\right)}}\left[P_{m}\right]\right)\right],
$$

where $Y=F\left(P_{n}\left[P_{m}\right]\right)$ index values, $b_{0}$ is the constant, $b_{1}$ is the regression coefficient, and $X=F\left(P_{n_{\left(Q_{\alpha}\right)}}\left[P_{m}\right]\right)$ index values (see Tables 1 and 2).

$$
Y=11263.1338+0.00008912 X \text {. }
$$

3.5. Hyper Zagreb Index.

$$
H M\left(P_{n}\left[P_{m}\right]\right)=b_{0}+b_{1}\left[H M\left(P_{n_{\left(Q_{\alpha}\right)}}\left[P_{m}\right]\right)\right]
$$

where $Y=H M\left(P_{n}\left[P_{m}\right]\right)$ index values, $b_{0}$ is the constant, $b_{1}$ is the regression coefficient, and $X=H M\left(P_{n_{\left(Q_{\alpha}\right)}}\left[P_{m}\right]\right)$ index values (see Tables 1 and 2).

$$
Y=30210.7664+0.0002023 X \text {. }
$$

Now, we represent a tabular representation of the correlation and square of correlation coefficient values related to the TI's of certain type of graphs $P_{n_{\left(Q_{\alpha}\right)}}\left[P_{m}\right]$ and $P_{n}\left[P_{m}\right]$ (see Tables 3 and 4 ). The highest value of $R$ and $R^{2}$ are indicated in bold.

For more explanation, see the graphical representation of correlation and square of correlation coefficient in Figures 5 and 6 .

\section{Data Availability}

All the data are included within this paper. However, the reader may contact the corresponding author for more details of the data. 


\section{Conflicts of Interest}

The authors have no conflicts of interest.

\section{References}

[1] I. Gutman and N. Trinajstić, "Graph theory and molecular orbitals. Total $\varphi$-electron energy of alternant hydrocarbons," Chemical Physics Letters, vol. 17, no. 4, pp. 535-538, 1972.

[2] X. Li and J. Zheng, "A unified approach to the extremal trees for different indices," MATCH Communications in Mathematical and in Computer Chemistry, vol. 54, pp. 195-208, 2005.

[3] B. Furtula and I. Gutman, "A forgotten topological index," Journal of Mathematical Chemistry, vol. 53, no. 4, pp. 11841190, 2015.

[4] G. H. Shirdel, H. Rezapour, and A. M. Sayadi, "Hyper-Zagreb index of graph operations," Iranian Journal of Mathematical Chemistry, vol. 4, pp. 213-220, 2013.

[5] I. Gutman and O. Polansky, Mathematical Concepts in Organic Chemistry, Springer-Verlag, Berlin, Germany, 1986.

[6] M. R. F. M. Rezaiel, W. Gao, and M. K. Siddiqui, "Computing hyper zagreb index and $\mathrm{m}$-polynomials of titania nanotubes tio2[m,n]," Sigma Journal of Engineering and Natural Sciences, vol. 35, no. 4, pp. 707-714, 2016.

[7] M. K. Siddiqui, M. Imran, and A. Ahmad, "On Zagreb indices, Zagreb polynomials of some nanostar dendrimers," Applied Mathematics and Computation, vol. 280, pp. 132-139, 2016.

[8] A. N. A. Koam, A. Ahmad, and A. A. Ahamdini, "Computation of vertex-edge degree based topological descriptors for hex-derived networks," IEEE Access, vol. 9, pp. 82989-83001, 2021.

[9] A. Iqbal, G. Ali, J. Khan, M. N. G. Rahmat, and A. Kausar, "On topological indices of dual graphs of Benzene ring embebded in P-type surface in 2D-Network," International Journal of Advanced Trends in Computer Science and Engineering, vol. 10, no. 3, pp. 1936-1941, 2021.

[10] U. Ahmad and S. Hameed, "Study of topological indices in a class of benzenoid graphs," Computational Journal of Combinatorial Mathematics, vol. 1, pp. 19-30, 2020.

[11] M. Asif and M. Hussain, "Topological characterization of cellulose network," Computational Journal of Combinatorial Mathematics, vol. 2, pp. 21-30, 2020.

[12] R. Todeschini, V. Consonni, R. Mannhold, H. Kubinyi, and H. Timmerman, Handbook of Molecular Descriptors, Wiley VCH, Weinheim, Germany, 2002.

[13] W. Yan, B.-Y. Yang, and Y.-N. Yeh, "The behavior of Wiener indices and polynomials of graphs under five graph decorations," Applied Mathematics Letters, vol. 20, no. 3, pp. 290-295, 2007.

[14] J.-B. Liu, S. Javed, M. Javaid, and K. Shabbir, "Computing first general zagreb index of operations on graphs," IEEE Access, vol. 7, pp. 47494-47502, 2019.

[15] M. Eliasi and B. Taeri, "Four new sums of graphs and their Wiener indices," Discrete Applied Mathematics, vol. 157, no. 4, pp. 794-803, 2009.

[16] Y.-M. Chu, S. Javed, M. Javaid, and M. Kamran Siddiqui, "On bounds for topological descriptors of $\varphi$-sum graphs," Journal of Taibah University for Science, vol. 14, no. 1, pp. 1288-1301, 2020.

[17] H. Deng, D. Sarala, S. K. Ayyaswamy, and S. Balachandran, "The Zagreb indices of four operations on graphs," Applied Mathematics and Computation, vol. 275, pp. 422-431, 2016.
[18] S. Akhter and M. Imran, "Computing the forgotten topological index of four operations on graphs," AKCE International Journal of Graphs and Combinatorics, vol. 14, no. 1, pp. 70-79, 2017.

[19] J.-B. Liu, M. Javaid, and H. M. Awais, "Computing zagreb indices of the subdivision-related generalized operations of graphs," IEEE Access, vol. 7, pp. 105479-105488, 2019.

[20] M. Javaid, S. Javed, A. M. Alanazi, and M. R. Alotaibi, "Computing analysis of Zagreb indices for generalized sum graphs under strong product," Journal of Chemistry, vol. 2021, Article ID 6663624, 20 pages, 2021.

[21] D. Sarala, H. Deng, S. K. Ayyaswamy, and S. Balachandran, "The Zagreb indices of graphs based on four new operations related to the lexicographic product," Applied Mathematics and Computation, vol. 309, pp. 156-169, 2017.

[22] N. De, "F-index of graphs based on four operations related to the lexicographic product," 2017, https://arxiv.org/abs/1706. 00464.

[23] K. Pattabiraman, "Four new operations related to composition and their hyper-zagreb index," Southeast Asian Bulletin of Mathematics, vol. 43, no. 6, 2019.

[24] K. Pattabiraman and A. Santhakumar, "Four new operations related to composition and their reformulated Zagreb index," Indonesian Journal of Combinatorics, vol. 2, no. 1, pp. 35-49, 2018.

[25] M. Suresh and G. S. Devi, "Some operations in hyper Zagreb indices," AIP Conference Proceedings, vol. 2277, no. 1, Article ID 140003, 2020.

[26] M. Javaid, S. Javed, S. Q. Memon, and A. M. Alanazi, "Forgotten index of generalized operations on graphs," Journal of Chemistry, vol. 2021, Article ID 9971277, 14 pages, 2021.

[27] D. A. Alrowaili, S. Javed, and M. Javaid, "On the exact values of HZ-index for the graphs under operations," Journal of Mathematics, vol. 2021, Article ID 3304939, 17 pages, 2021.

[28] M. Javaid, S. Javed, Y. F. Alanazi, and A. M. Alanazi, "Computing correlation among the graphs under lexicographic product via zagreb indices," Journal of Chemistry, vol. 2021, Article ID 7465171, 17 pages, 2021.

[29] Z. B. Peng, S. Javed, M. Javaid, and J. B. Liu, "Computing FGZ index of sum graphs under strong product," Journal of Mathematics, vol. 2021, Article ID 6654228, 16 pages, 2021. 